JPHSR 2010, 1: 137-141

(c) 2010 The Authors JPHSR @ 2010 Royal Pharmaceutical Society Received June 6, 2010 Accepted July 22, 2010 DOI

10.1111/j.1759-8893.2010.00020.x ISSN 1759-8885
Correspondence: Anders Ekedahl, clo Apoteket Lejonet, Stortorget 8, SE-211 34 Malmö, Sweden.

E-mail: anders.ekedahl@Inu.se

\section{Dosages involving splitting tablets: common but unnecessary?}

\author{
Christer Berg ${ }^{\mathrm{a}, \mathrm{b}}$ and Anders Ekedah $\mathrm{l}^{\mathrm{b}, \mathrm{c}}$
}

aNational Corporation of Swedish Pharmacies, Växjö, bSchool of Natural Sciences, Linnæus University, Kalmar and ${ }^{C} R \& D$ Department, National Corporation of Swedish Pharmacies, Malmö, Sweden

\begin{abstract}
Objectives Prescribing of treatments with dosages involving split tablets is common. Many patients report they have difficulties in dividing the tablets and in following the prescribed treatment. The objective of this study was to examine to what extent dosages involving split tablets are prescribed in Sweden.

Methods The dosage text strings were analysed on prescriptions dispensed during one month at Swedish pharmacies on all tablet formulations for beta-blockers, calcium blockers, angiotensin-converting enzyme inhibitors, angiotensin receptor blockers (ARBs), lipidlowering agents, levothyroxine, neuroleptics, anxiolytics, hypnotics/sedatives and selective serotonin reuptake inhibitors (SSRIs). Numbers and percentages of prescriptions with split tablets were compared with all dispensed prescriptions.
\end{abstract}

Key findings Six hundred thousand prescriptions on tablet formulations for the investigated drugs were dispensed. Ten per cent of the prescriptions had a dosage where tablets have to be split. Hypnotics and SSRIs had the highest proportions, accounting for 22 and $19 \%$ of prescriptions involving split tablets. SSRIs constituted $30 \%$ of the prescriptions with split tablets. Dosages with split tablets varied with drug across patient age but not across gender. In $45 \%$ of the cases with levothyroxine and SSRIs, a tablet strength fitting the prescribed dosage was licensed and available. Furthermore, it would have been possible to avoid splitting tablets by adjusting and combining existing licensed strengths for more than $80 \%$ of the prescriptions.

Conclusions Prescribing of dosages involving the splitting of tablets was common and constituted $10 \%$ of the prescriptions for tablet formulations. Many prescriptions on dosages with split tablets can be avoided if physicians adjust prescribing to licensed and available strengths fitting the prescribed dosages.

Keywords drug-related problem; pharmacy; prescriptions; split tablets; Sweden

\section{Introduction}

Prescribing of dosages involving split tablets is common, and even more so among elderly patients and those with several medications. ${ }^{[1-4]}$ Difficulties in dividing tablets are also common: one- to two-thirds of patients with prescribed dosages involving split tablets state they have difficulties in dividing the tablets. ${ }^{[2-9]}$ Elderly patients in particular find it difficult and may fail or are unable to split tablets due to vision problems or motor impairment. Concerns have been raised that tablet splitting may compromise patient safety as well as clinical efficacy ${ }^{[10,11]}$ Many tablets are not designed to be split or are difficult to divide, and tablets may break into uneven parts or crumble. The European Pharmacopoeia accepts a dosage accuracy of the individual parts of the tablet within $\pm 15 \%$ of the anticipated dose. However, accuracy when splitting tablets is low and the fluctuation in dose may be large. ${ }^{[12-17]}$ To facilitate splitting of tablets the use of a tablet splitter is recommended, but for a variety of reasons few patients use them. ${ }^{[2,4]}$ The cost of the tablet splitter may be judged too high, the tablet splitter may be difficult to handle and patients report they have not received any or adequate handling instructions. ${ }^{[4,6,18]}$ Moreover, the accuracy of tablet splitters in dividing tablets into equal parts often produces halves no better than produced by manual splitting with an unacceptably large deviation from the intended dose. ${ }^{[12,19]}$ (other strengths or other administration forms) are available to be prescribed instead..$^{[4]}$ Dosages with split tablets may be prescribed for a number of reasons, which may vary by 
drug. Due to pharmacokinetic variation among individuals the standardised dose may give rise to a variable response between individual patients; therefore the dose must be individually selected. Children, adolescents and elderly patients often need lower doses. For many brands, few tablet strengths are available and those available may not correspond to the selected dosage. The most common single dose strengths may not be licensed and, furthermore, pharmaceutical companies may be unaware of how their drugs are prescribed. Renewals of prescriptions to patients on chronic medication may be done without adjustment to available strengths. Patients may have trouble swallowing whole tablets. Prescribing of tablets of twice the required strength that are intended to be split may be recommended for cost-containment reasons or even be mandatory for reimbursement, especially for new drugs under patent protection. ${ }^{[18-26]}$ Patients may also prefer to receive prescriptions for tablets of higher strength and to split tablets in order to decrease their out-of-pocket costs. ${ }^{[19,27]}$ There may be other reasons as well. For instance, in Sweden, split tablets may not be subject to generic substitution and prescriptions for split tablets of drugs with a cost above the threshold level may be reimbursed. However, the pharmacokinetic profile of the drug may be altered when tablets not intended to be divided are cut in half; the effect may be different from anticipated, which may increase the incidence of adverse drug reactions.

The present study was performed to examine to what extent dosages with split tablets are prescribed in Sweden and if alternative strengths or administration forms are available.

\section{Methods}

The setting was Swedish pharmacies. Data on all dispensed prescriptions at Swedish pharmacies, including the dosage text string on the prescription, are stored in the pharmacy transaction database, Apotekets TransaktionsDatabas (ATD).

A cross-sectional study was performed. All prescriptions for tablet formulations dispensed at Swedish pharmacies during one month, October 2004 or October 2005, for selected Anatomical Therapeutic Chemical (ATC) groups, were extracted from ATD. The extracted data included patient gender and year of birth, dispensed drug (ATC code, name, administration formula, strength, pack size) and the dosage text string.

Inclusion criteria were prescriptions made to humans of the following drugs. In October 2004: ATC groups C07 (beta-blockers), C08 (calcium blockers), N05CD (hypnotics/ sedatives) and N06AB (selective serotonin reuptake inhibitors (SSRIs)); in October 2005: ATC groups C09 (angiotensinconverting enzyme (ACE) inhibitors and angiotensin receptor blockers, ARBs), C10 (lipid-lowering agents), H03 (levothyroxine), N05A (neuroleptics) and N05BD (anxiolytics). Only drugs administered as tablets were included. Prescriptions made to animals were excluded.

The main outcome measures were the numbers and percentages of prescriptions with split tablets compared with all dispensed prescriptions.

The data were placed in a Microsoft Excel spreadsheet for calculations. Twelve undergraduate students were trained and analysed one drug group each using the same method/ protocol.

\section{Results}

We examined 607794 prescriptions for 74 different substances in nine ATC groups (see Table 1). Dosages with split tablets were prescribed in $9.7 \%$ of all prescriptions for tablet formulas (range $0 ; 34 \%$ ). Split tablets were somewhat more prevalent in prescriptions to women than men (12.1 and $10.3 \%$, see Figure 1), and there were differences across age; however, the proportions of prescriptions on split tablets varied with type of drug.

Hypnotics and SSRIs had the highest proportions of prescriptions on dosages where tablets had to be split: 21.9 and $19.2 \%$ respectively. Prescriptions for SSRIs and beta-blockers constituted $53 \%$ (31 and 22\%, respectively) of all prescriptions on dosages with split tablets. Sixteen substances with more than 1000 prescriptions as split tablets $(n=51987$ of 385513 ) constituted $64 \%$ of the dispensed prescriptions and $88 \%$ of the prescriptions with split tablets (see Table 2). For 19 substances, more than $15 \%$ of all prescriptions were using split tablets (mean $\pm \mathrm{SD}, 15.3 \pm 7.1 \%$; median, $16.7 \%$ ) and

Table 1 Proportions of prescriptions with dosages with split tablets in Sweden for 74 studied substances in nine ATC groups of drugs $(n=607794)$

\begin{tabular}{|c|c|c|c|c|c|c|c|}
\hline \multirow[t]{2}{*}{ ATC code } & \multirow{2}{*}{$\begin{array}{l}\text { Drug group (number of } \\
\text { substances) }\end{array}$} & \multirow{2}{*}{$\begin{array}{c}\text { Dispensed prescriptions } \\
n\end{array}$} & \multicolumn{5}{|c|}{ Prescriptions with split tablets } \\
\hline & & & $n$ & $\%$ & Mean \pm SD & Median & Range \\
\hline $\mathrm{C} 07$ & Beta blockers (10) & 148995 & 12798 & 8.6 & $10.1 \pm 7.1$ & 8.9 & $0.0-20.4$ \\
\hline $\mathrm{C} 08$ & Calcium blockers $(8)$ & 39858 & 868 & 2.2 & $2.2 \pm 2.0$ & 2.0 & $0.0-5.5$ \\
\hline C09 & ACE inhibitors (7)/ARBs (5) & 84447 & 5673 & 6.7 & $4.5 \pm 2.5$ & 3.8 & $0.6-8.6$ \\
\hline $\mathrm{C} 10$ & Lipid-lowering agents (10) & 115154 & 1319 & 1.1 & $1.8 \pm 3.2$ & 0.7 & $0.0-10.0$ \\
\hline H03 & Levothyroxine (1) & 44755 & 7780 & 17.4 & $(17.4)$ & $(17.4)$ & $(17.4)$ \\
\hline N05A & Neuroleptics (20) & 31288 & 2188 & 7.0 & $6.6 \pm 9.8$ & 2.1 & $0.0-33.7$ \\
\hline N05BA & Anxiolytics (4) & 39942 & 5935 & 14.9 & $12.7 \pm 9.5$ & 14.2 & $0.0-22.2$ \\
\hline N05CD & Sedatives/hypnotics (3) & 17573 & 3995 & 22.7 & $22.3 \pm 3.2$ & 21.9 & $19.3-25.7$ \\
\hline N06AB & Antidepressants/SSRIs (6) & 85785 & 18367 & 21.4 & $19.7 \pm 5.1$ & 19.2 & $13.7-27.9$ \\
\hline Total & 74 & 607794 & 58924 & 9.7 & $11.3 \pm 8.0$ & 8.6 & $1.8-22.3$ \\
\hline
\end{tabular}

ACE, angiotensin-converting enzyme; ARB, angiotensin receptor blocker; ATC, Anatomical Therapeutic Chemical classification; SSRI, selective serotonin reuptake inhibitor. 

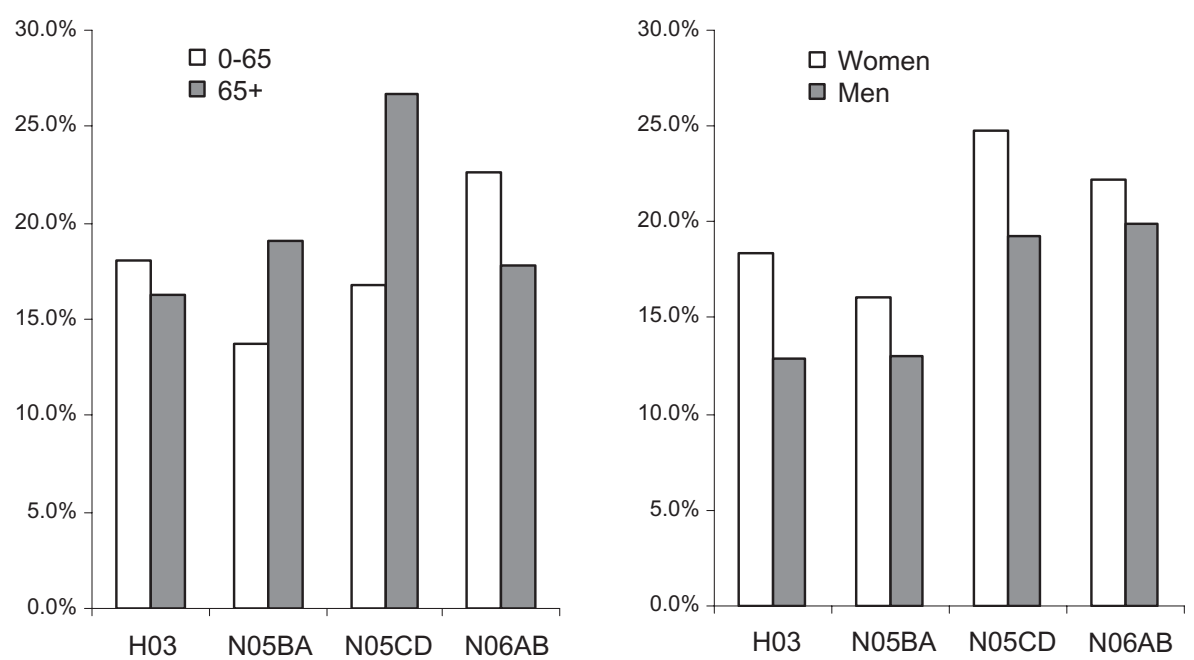

Figure 1 Proportions of prescriptions for split tablets in Sweden with reference to age (left) and gender (right) for levothyroxine (H03, $n=44755$ ), anxiolytics (N05BA; $n=39$ 942), sedatives/hypnotics (N05CD; $n=17573)$ and selective serotonin reuptake inhibitors (N06AB; $n=85785)$.

Table 2 Dispensed prescriptions for 16 drugs with more than 1000 prescriptions with split tablets $(n=51978)$, constituting $88.2 \%$ of all prescriptions for split tablets and $63.4 \%$ (385 513) of all studied prescriptions

\begin{tabular}{llcrc}
\hline ATC code & Drug group & $\begin{array}{c}\text { Prescriptions with split } \\
\text { tablets }\end{array}$ & $\begin{array}{c}\text { All prescriptions } \\
\text { (numbers) }\end{array}$ & $\begin{array}{c}\text { Proportion of prescriptions } \\
\text { with split tablets (\%) }\end{array}$ \\
\hline N06AB05 & Paroxetin & 2488 & 8906 & 27.9 \\
N05CD03 & Flunitrazepam & 2302 & 8954 & 25.7 \\
N06AB04 & Citalopram & 8440 & 37114 & 22.7 \\
C07AA07 & Sotalol & 1567 & 7666 & 20.4 \\
N06AB06 & Sertralin & 5374 & 26425 & 20.3 \\
N05CD02 & Nitrazepam & 1438 & 7455 & 19.3 \\
H03AA01 & Levothyroxine & 7780 & 44755 & 17.4 \\
N05BA01 & Diazepam & 4175 & 24981 & 16.7 \\
C07AB07 & Bisoprolol & 1726 & 10561 & 16.3 \\
N06AB10 & Escitalopram & 1046 & 7619 & 13.7 \\
C07AA05 & Propranolol & 1289 & 10193 & 12.6 \\
N05BA12 & Alprazolam & 1748 & 14904 & 11.7 \\
C07AB02 & Metoprolol & 4854 & 54263 & 8.9 \\
C09AA02 & Enalapril & 3692 & 48728 & 7.6 \\
C09AA05 & Ramipril & 1268 & 18021 & 5.0 \\
C07AB03 & Atenolol & 2791 & 54968 & 13.5 \\
Total & & 51978 & 385513 & \\
\hline
\end{tabular}

constituted $92.8 \%$ of all investigated prescriptions with dosages on split tablets. Of prescriptions with split tablets, $41 \%$ for cardiovascular drugs were to patients of 70 years or more compared with $21 \%$ for SSRIs.

Levothyroxine and SSRIs were studied separately. For $44.7 \%$ a tablet strength fitting the prescribed dosage was available. Avoiding use of split tablets would have been possible in more than $80 \%$ (95.3 and $81.8 \%$, respectively) of instances had existing licensed tablet strengths been used or combined.

\section{Discussion}

Our study shows that prescribing of dosages with split tablets is common in Sweden, especially for psychotropic drugs. If licensed and available strengths had been prescribed in the first place for levothyroxine and SSRIs, four out of five prescriptions for split tablets could have been avoided.

There are certain limitations to the study. The point of measure is the prescription and the only information on patients is gender and age but there are no individual data, which would have made cross-linking with other databases (outcome data) possible. The prescriptions studied were collected from two different periods and are thus not truly crosssectional. However, the drugs studied are usually used continuously. Overall results may not be representative for all other drugs, and the two subgroups of drugs we examined to see if there were tablet strengths that fitted the prescribed dosage (levothyroxine and SSRIs) may not have been representative of the other drugs. However, these two subgroups, 
with a high prevalence of prescriptions for split tablets, constituted a large part (44\%) of all studied prescriptions for dosages involving split tablets.

The prevalence of prescriptions with split tablets was higher in previous studies than in our study, 20 to more than $35 \%,{ }^{[1,2,4,7,28]}$ and the prevalence of elderly patients with at least one prescription requiring tablet splitting varied from 35 to $67 \% .^{[1,3]}$ Rodenhuis et al. found that for $46 \%$ of the prescriptions with tablet splitting, alternatives (tablets with half or quarter strength, or oral solution) were available, ${ }^{[7]}$ similar to our findings. However, many of previous studies were small and may not have been representative.

There are conflicting data on the impact of tablet splitting on patient adherence to prescribed therapy: some studies indicate that tablet splitting increases non-adherence ${ }^{[26,29]}$ as well as medication errors, ${ }^{[26]}$ and others state no impact on adherence or clinical outcome. ${ }^{[18,19,27,30,31]}$ Most studies are with selected patient groups and splitting is often accompanied by an economic incentive (the out-of-pocket co-payment for the patient may be decreased substantially). However, as elderly patients use many medications and are frailer and subject to visual and motor impairment, it is reasonable to assume that every addition to regimen complexity will increase the risk for non-adherence and medication errors as well as adverse drug reactions. ${ }^{[29]}$

Prescriptions with dosages requiring that tablets be split and patients' difficulty in splitting the tablets are common; ${ }^{[4-7]}$ so common that this may be overlooked by pharmacists. However, difficulties in splitting tablets are simple to identify and in many instances easy to solve. Pharmacists should be encouraged to ask patients how they manage to adhere to the prescribed treatment. If a patient has difficulty in splitting tablets, there may be a licensed strength or other administration formula to fit the dosage, or it may be possible to adjust the dosing schedule without compromising the efficacy before suggesting that the patient use a tablet splitter, followed by adequate instruction in its use.

Our study constitutes an analysis of more than 600000 prescriptions for tablet formulations, covering all prescriptions made in Sweden dispensed during one month for the selected drug groups. The Swedish pharmacy benefit scheme (PBS) allows a maximum of 90 days'/3 months' treatment to be dispensed with reimbursement at each fill. Accordingly, patients with continuous medication will have a refill every third month. Our results imply that at least 180000 prescriptions to more than 125000 patients on continuous medication in Sweden have a dosage involving split tablets, corresponding to at least 40000 patients on continuous medication having difficulties in splitting the tablets and adhering to the prescribed treatment.

The results of this study suggest that (a) pharmacists should be permitted to adjust dispensing to a strength that fits with the prescribed dosage level, (b) price policies with flat prices need to be revised to eliminate economic incentives to prescribe dosages with split tablets and (c) physicians, pharmacists and patients should demand that the pharmaceutical companies license tablet strengths that fit the prescribed dosages. A decrease in the use of split tablets would result in both an increased ability for patients to comply with the prescribed therapy and a decrease in changes in clinical effect and adverse drug reactions due to unpredicted pharmacokinetic differences in the preparations.

In future studies we aim to interview an extended group of patients on how they manage to split tablets and follow the prescribed treatment and to observe what information pharmacists give patients with prescriptions for split tablets.

\section{Conclusions}

Prescribing in Sweden of dosages involving split tablets is common and constituted $10 \%$ of the prescriptions examined in the present study. Dosages with split tablets can be avoided if physicians adjust prescribing to licensed and available strengths fitting the prescribed dosages.

\section{Declarations}

\section{Conflict of interest}

The Author(s) declare(s) that they have no conflicts of interest to disclose.

\section{Funding}

This research received no specific grant from any funding agency in the public, commercial, or not-for-profit sectors.

\section{Acknowledgements}

We gratefully acknowledge the students Ida Bäckman, Diana Johansson, Johanna Johansson, Sara Lehtonen, Johanna Lindberg, Kristin Lindholm, Marielle Lundholm, Anna Nilsson, Anna Törnquist, Elisabeth Utbult, Fabian Westlund and Jenny Wiklander for their participation and Hans Moosberg for help with extracting the data from ATB.

\section{References}

1. Fischbach MS et al. Pill-splitting in a long-term care facility. Can Med Assoc J 2001; 164: 785-786.

2. Quinzler R et al. The frequency of inappropriate tablet splitting in primary care. Eur J Clin Pharmacol 2006; 62: 1065-1073.

3. Denneboom W et al. User-related pharmaceutical care problems and factors affecting them: the importance of clinical relevance. J Clin Pharm Ther 2005; 30: 215-223.

4. Rodenhuis $\mathrm{N}$ et al. Patient experiences with the performance of tablet score lines needed for dosing. Pharm World Sci 2003; 25 : 173-176.

5. Atkin PA et al. Functional ability of patients to manage medication packaging: a survey of geriatric inpatients. Age Ageing 1994; 23: 113-116.

6. Quinzler R et al. Tablet splitting: patients and physicians need better support. Eur J Clin Pharmacol 2007; 63: 1203-1204.

7. Rodenhuis $\mathrm{N}$ et al. The rationale of scored tablets as dosage form. Eur J Pharm Sci 2004; 21: 305-308.

8. Dunning T, Manias E. Medication knowledge and selfmanagement by people with type 2 diabetes. Aust J Adv Nurs 2005; 23: 7-14.

9. Wilson MG et al. Tablet-breaking ability of older persons with type 2 diabetes mellitus. Diabetes Educ 2001; 27: 530-540.

10. Sales MM, Cunningham FE. Tablet splitting. Top Patient Saf 2006; 6: 1, 4. 
11. Hixson-Wallace JA et al. Effect of regimen complexity on patient satisfaction and compliance with warfarin therapy. Clin Appl Thromb Hemost 2001; 7: 33-37.

12. Peek BT et al. Accuracy of tablet splitting by elderly patients. JAMA 2002; 288: 451-452.

13. Matuschka PR, Graves JB. Mean dose after splitting sertraline tablets. J Clin Psychiatry 2001; 62: 826.

14. McDevitt JT et al. Accuracy of tablet splitting. Pharmacotherapy 1998; 18: 193-197.

15. Rosenberg JM et al. Weight variability of pharmacist-dispensed split tablets. J Am Pharm Assoc (Wash) 2002; 42: 200-205.

16. Teng $\mathrm{J}$ et al. Lack of medication dose uniformity in commonly split tablets. J Am Pharm Assoc (Wash) 2002; 42: 195199.

17. Stimpel M et al. The scored tablet - a source of error in drug dosing? J Hypertens Suppl 1985; 3: S97-S99.

18. Fawell NG et al. Relationship between tablet splitting and compliance, drug acquisition cost, and patient acceptance. Am J Health Syst Pharm 1999; 56: 2542-2545.

19. Choe HM et al. Impact of patient financial incentives on participation and outcomes in a statin pill-splitting program. Am J Manag Care 2007; 13: 298-304.

20. Cohen CI, Cohen SI. Potential cost savings from pill splitting of newer psychotropic medications. Psychiatr Serv 2000; 51: 527529.

21. Dobscha SK et al. Strategies to decrease costs of prescribing selective serotonin reuptake inhibitors at a VA Medical Center. Psychiatr Serv 2003; 54: 195-200.
22. Stafford RS, Radley DC. The potential of pill splitting to achieve cost savings. Am J Manag Care 2002; 8: 706-712.

23. Hamer AM et al. Initial results of the use of prescription order change forms to achieve dose form optimization (consolidation and tablet splitting) of SSRI antidepressants in a state Medicaid program. J Manag Care Pharm 2006; 12: 449-456.

24. Vuchetich PJ et al. Evaluation of cost savings to a state medicaid program following a sertraline tablet-splitting program. $J A m$ Pharm Assoc (Wash) 2003; 43: 497-502.

25. Schellhase EM, Hardin AK. Development and implementation of a tablet splitting education program in a veterans affairs medical center. Hosp Pharm 2003; 38: 453-457.

26. Gee $\mathrm{M}$ et al. Effects of a tablet-splitting program in patients taking HMG-CoA reductase inhibitors: analysis of clinical effects, patient satisfaction, compliance, and cost avoidance. $J$ Manag Care Pharm 2002; 8: 453-458.

27. Rindone JP. Evaluation of tablet-splitting in patients taking Lisinopril for hypertension. J Clin Outcomes Manag 2000; 7: 22-24.

28. Stankovic I et al. Breaking of tablets as a cost-saving strategy. $J$ Reg Sect Serb Med Assoc 2007; 32: 5-10.

29. Odegard PS, Capoccia K. Medication taking and diabetes: a systematic review of the literature. Diabetes Educ 2007; 33: 1014-1029.

30. Duncan MC et al. Effect of tablet splitting on serum cholesterol concentrations. Ann Pharmacother 2002; 36: 205-209.

31. Parra D et al. Effect of splitting simvastatin tablets for control of low-density lipoprotein cholesterol. Am J Cardiol 2005; 95: 1481-1483. 